Sports Biomechanics

Original article

The correlation between jump height and mechanical power in a countermovement jump is artificially inflated

Nicholas P. Linthorne

Division of Sport, Health and Exercise Sciences, Department of Life Sciences, Brunel University London, Uxbridge, United Kingdom

Author contact details:

Nicholas P. Linthorne

Division of Sport, Health and Exercise Sciences

Department of Life Sciences

Brunel University London

Uxbridge, Middlesex, UB8 3PH

United Kingdom

Email: nick.linthorne@brunel.ac.uk

ORCID: https://orcid.org/0000-0001-9709-5955 


\title{
The correlation between jump height and mechanical power in a countermovement jump is artificially inflated
}

\author{
The countermovement jump is commonly used to assess an athlete's \\ neuromuscular capacity. The aim of this study was to identify the mechanism \\ behind the strong correlation between jump height and mechanical power in a \\ countermovement jump. Three athletes each performed between 47 and 60 \\ maximal-effort countermovement jumps on a force platform. For all three \\ athletes, peak mechanical power and average mechanical power were strongly \\ correlated with jump height $(r=0.54-0.90)$. The correlation between jump height \\ and peak power was largely determined by the correlation between jump height \\ and the velocity at peak power $(r=0.83-0.94)$ and was not related to the \\ correlation between jump height and the ground reaction force at peak power \\ $(r=-0.20-0.18)$. These results confirm that the strong correlation between jump \\ height and power is an artefact arising from how power is calculated. Power is a \\ compound variable calculated from the product of instantaneous ground reaction \\ force and instantaneous velocity, and application of statistical theory shows that \\ the correlation between jump height and power is artificially inflated by the near- \\ perfect correlation between jump height and the velocity at peak power. Despite \\ this finding, mechanical power might still be useful in assessing the \\ neuromuscular capacity of an athlete.
}

Keywords: athlete monitoring; neuromuscular power; single-subject analysis; vertical jump

\section{Introduction}

Many high-performance sport programs make use of regular performance tests to help guide the athlete's training schedule and competition schedule. The countermovement jump is one of the most common tests for assessing the neuromuscular capacity of an athlete's lower body, and the most accurate method for obtaining jump data is with a force platform (McGuigan, 2017; McMahon, Lake, \& Suchomel, 2018). Highresolution measurements of the vertical ground reaction force from a force platform can be used to calculate a large number of variables including the jump height, the peak 
force, the rate of force development, the depth of countermovement, and the durations of the phases of the jump. Force platform data can also be used to calculate the instantaneous mechanical power flow to the ground during the jump. Mechanical power is usually reported as the peak mechanical power and the time-average mechanical power during the upward propulsion phase of the jump. However, several commentators have highlighted issues with using mechanical power in a jump to assess neuromuscular capacity (Adamson \& Whitney, 1971; Knudson, 2009; Winter, 2005). The mechanical power flow to the ground is not the same as the physiological power generated by the jumper's muscles (Morin, Jiménez-Reyes, Brughelli, \& Samozino, 2019). Although most of the power generated by the muscles during the upward propulsion phase of the jump contributes to increasing the potential energy and kinetic energy of the jumper's centre of mass, some muscle power is used in rotating the body segments and so does not contribute to the jump height (Bobbert, 2014; Bobbert and Van Soest, 2001). Also, a clear theoretical link between mechanical power flow to the ground and jump height has not been established; it is not clear how changes in peak mechanical power and average mechanical power are related to changes in jump height.

Despite these issues, many studies of vertical jumping have measured mechanical power and several studies have reported a strong correlation between mechanical power and jump height (Aragón-Vargas \& Gross, 1997; Barker, Hart, \& Mercer, 2017; Dowling \&Vamos, 1993; Harman, Rosenstein, Frykman, \& Rosenstein, 1990; Markovic, Mirkov, Nedeljkovic, \& Jaric, 2014). The rationale for reporting mechanical power in an athlete performance test appears to be that if mechanical power is strongly correlated with jump height then mechanical power might be a performancelimiting factor in a countermovement jump (Kennedy \& Drake, 2018; Lake \& Mundy, 2018). 
Here, it is argued that the strong correlation between jump height and mechanical power is an artefact arising from how power is calculated. To understand the rationale for this argument we first need to review the time traces of position, velocity, ground reaction force, and power in a typical countermovement jump (Figure 1). Before commencing the jump there is stationary phase where the ground reaction force is equal to the jumper's body weight and the position and velocity are zero. The jumper then has a downward countermovement phase, an upward propulsion phase, a flight phase, and a landing phase. The velocity is zero at the bottom of the countermovement and reaches a maximum shortly before take-off (Linthorne, 2001). During the upward propulsion phase the velocity increases almost linearly with time. Also, the ground reaction force remains high throughout most of the upward propulsion phase but decreases rapidly to zero just before take-off. Instantaneous power is the product of the ground reaction force and velocity, and so instantaneous power increases almost linearly with time during the upward propulsion phase. Peak instantaneous power occurs shortly before take-off, just as the ground reaction force starts to rapidly decrease.

[Figure 1 near here]

There are two key features that could have a profound influence on the strength of the correlation between jump height and mechanical power. The first is that power is a compound variable calculated as the product of two other variables (force and velocity). Statisticians have derived expressions that allow us to calculate a correlation coefficient when the output variable is the product of two other variables (Appendix). The main result is that the strength of the correlation between jump height and peak power $\left(\rho_{H, P}\right)$ is given by a sum of the correlation between jump height and the ground reaction force at peak power $\left(\rho_{H, F}\right)$ and the correlation between jump height and the 
velocity at peak power $\left(\rho_{H, V}\right)$, with a small effect from the correlation between the ground reaction force at peak power and the velocity at peak power $\left(\rho_{F, V}\right)$;

$$
\rho_{H, P} \approx \frac{a}{\sqrt{a^{2}+b^{2}+c\left(\rho_{F, V}\right)}} \rho_{H, V}+\frac{b}{\sqrt{a^{2}+b^{2}+c\left(\rho_{F, V}\right)}} \rho_{H, F}
$$

where $a, b$, and $c$ are constants specific to the jumper.

The second key feature is that in a countermovement jump the correlation between jump height and the velocity at peak power $\left(\rho_{H, V}\right)$ should be almost perfect. To see why this should be so we note that jump height is calculated from the take-off velocity ( $h=v_{\mathrm{to}}{ }^{2} / 2 g$, where $g$ is the acceleration due to gravity) and so the correlation between jump height and take-off velocity is perfect (i.e., exactly 1). Also, for a substantial portion of the upward propulsion phase there is a near-linear relationship between velocity and position (Figure 2), and so take-off velocity is almost perfectly correlated with the velocity at any given position. Therefore, jump height should be almost perfectly correlated with the velocity at the position of peak power.

[Figure 2 near here]

The combined effect of these two key features is that jump height is expected to have a strong correlation with peak power (Appendix). The strength of the correlation is artificially inflated by the near-perfect correlation between jump height and the velocity at peak power $\left(\rho_{H, V}\right)$.

Jump height should also be strongly correlated with the average power during the upward propulsion phase. During the upward propulsion phase the time-average power is about equal to half the peak power, the time-average ground reaction force is about equal to the peak ground reaction force, and the time-average velocity is about equal to half the take-off velocity (Figure 1). However, average power is not calculated as the product of average force and average velocity. Rather, average power is 
calculated by numerically integrating the instantaneous power over the duration of the upward propulsion phase. Even so, average power should be almost perfectly correlated with peak power and so jump height is expected to be strongly correlated with average power.

If the above arguments are correct the correlation between jump height and mechanical power in a set of jumps by an athlete will be artificially inflated. An artificially high correlation might be misleading about which variables should be monitored when assessing an athlete. Sport scientists and trainers might be placing undue emphasis on a variable (mechanical power) that does not have a strong causal influence on the athlete's jump height or does not clearly reflect changes in the athlete's neuromuscular performance (Morin et al, 2019).

The aims of the present study were to measure the correlation between jump height and mechanical power in a set of countermovement jumps by an athlete and to identify the mechanism that determines the strength of the correlation. The hypothesis was that peak power and average power in a countermovement jump are strongly correlated with jump height and that the mechanism for the strong correlation is that power is a compound variable calculated as the product of ground reaction force and velocity. Three sets of evidence were proposed that would support the hypothesis: 1) The correlation between jump height and peak power calculated using the statistical theory will be in close agreement with the observed correlation; 2) The observed correlation between jump height and the velocity at peak power will be almost perfect; and 3) The observed correlation between jump height and peak power will be largely determined by the correlation between jump height and the velocity at peak power, and will not necessarily be related to the correlation between jump height and the ground 
reaction force at peak power or to the correlation between the ground reaction force at peak power and the velocity at peak power.

\section{Methods}

This study used an experimental design with a single-subject analysis in which the data from each participant was analysed separately (Bates, 1996; Bates, James, \& Dufek, 2004). Three participants each performed a large number of maximal-effort countermovement jumps on a force platform. The outcome variable was the jump height and the main predictor variables were the peak power in the upward propulsion phase, the ground reaction force at peak power, the velocity at peak power, and the average power in the upward propulsion phase. The correlation coefficients for the relations between jump height and the predictor variables were calculated and compared.

Simulated jump data were also generated using a simple mathematical model of the upward propulsion phase of a countermovement jump. The simulated jump data were generated to help generalise the findings from the participants to other athletes. If the findings from the simulated jump data are similar to those from the experimental jump data this would indicate there are physical mechanisms that underlie the observed correlations in the participants. Therefore, other athletes would be expected to show correlations similar to those observed in the participants.

\section{Experimental jumps}

This study was conducted in accordance with the Declaration of Helsinki and the protocol was approved by the Ethics Committee of Brunel University London. One adult female football player and two collegiate male futsal players each performed between 47 and 60 maximal-effort countermovement jumps without arm swing on a force platform (Table 1 and Supplemental Online Material 1). The jump testing sessions 
took place in an indoor biomechanics laboratory using a $60 \times 40 \mathrm{~cm}$ Kistler piezoelectric force platform (type 9281B11; Kistler Instrumente, Winterthur, Switzerland). Participant 1 attended three jump testing sessions per day on four consecutive days, and Participants 2 and 3 attended five jump testing sessions per day on two consecutive days. The physical condition of the participants was nominally the same in all the test sessions. During the testing period the participants did not perform any strenuous physical activity, did not consume caffeine or alcohol, and maintained their usual diet. In each test session the participants performed up to seven maximaleffort countermovement jumps with at least one minute of rest between each jump. The participants were instructed to use a self-selected countermovement depth and to aim for maximum height in the flight phase of the jump. Arm movement was constrained by having the participants grip a lightweight aluminium rod that was positioned across their shoulders. Visual inspection during the test sessions indicated that the participants maintained a consistent jumping technique in all their jumps. Each participant used a similar rate of countermovement and countermovement depth in all their jumps and appeared to use a consistent sequence of extension of the hips, knees, and ankles in the upward propulsion phase.

[Table 1 near here]

Time traces of the vertical ground reaction force for the jumps were obtained using BioWare biomechanical analysis software (version 5.2; Kistler Instrumente, Winterthur, Switzerland) with the sampling rate set to $1000 \mathrm{~Hz}$. The BioWare software was used to obtain the depth of countermovement, the force at the bottom of the countermovement, the peak power in the upward propulsion phase, the ground reaction force at peak power, the position at peak power, the velocity at peak power, the average power in the upward propulsion phase, the average ground reaction force in the upward 
propulsion phase, the average velocity in the upward propulsion phase, the impulse in the upward propulsion phase, the duration of the upward propulsion phase, and the takeoff height. The take-off velocity and jump height were calculated using the impulsemomentum method (Linthorne, 2001). The estimated measurement uncertainties in the jump variables were: jump height, $0.001 \mathrm{~m}$; depth of countermovement, $0.005 \mathrm{~m}$; force at the bottom of the countermovement, $1 \mathrm{~N}$; take-off height, $0.007 \mathrm{~m}$; peak power, $9 \mathrm{~W}$; force at peak power, $2 \mathrm{~N}$; position at peak power, $0.007 \mathrm{~m}$; velocity at peak power, $0.010 \mathrm{~m} / \mathrm{s}$; average power, $5 \mathrm{~W}$; average force, $1 \mathrm{~N}$; and average velocity, $0.005 \mathrm{~m} / \mathrm{s}$ (Supplemental Online Material 1).

Each of the variables was tested for normality and screened for outliers using IBM SPSS Statistics v25 (IBM, Armonk, NY, USA). Scattergraphs of pairs of variables were created and the corresponding Pearson's correlation coefficients were calculated. The strength of the correlation was interpreted using the criteria proposed by Hopkins, Marshall, Batterham, and Hanin (2009). The threshold $r$ values for 'weak', 'moderate', 'strong', 'very strong', and 'near-perfect' correlations were $\pm 0.1,0.3,0.5,0.7$, and 0.9 , respectively.

\section{Simulated jumps}

Simulated jumps were generated using a simple model of the upward propulsion phase of a countermovement jump (Figure 3 ). In this model the three factors that determine the jump height are the depth of countermovement, the force at the bottom of the countermovement, and the shape of the force-position curve (Adamson \& Whitney, 1971). At the start of the jump the position of the jumper's centre of mass is zero and the ground reaction force is equal to the jumper's body weight $\left(F_{\mathrm{BW}}\right)$. The details of the downward countermovement phase are not relevant in this model, but at the bottom of the countermovement (i.e., the start of the upward propulsion phase) the jumper's centre 
of mass is at position $X_{\text {bottom }}$ and the ground reaction force is $F_{\text {bottom. In the upward }}$ propulsion phase the position profile of the ground reaction force is a power equation with exponent $A$. The shape of the force-position curve is mostly determined by the value of the exponent $A$ : a lesser value produces a curve that is more rounded and so the jumper performs less work; a greater value produces a curve that is more square-shaped and so the jumper performs more work. At the instant of take-off the ground reaction force is zero and the position of the jumper's centre of mass is $X_{\text {take-off }}$ above the zero position (because the jumper's ankles are plantarflexed at take-off).

[Figure 3 near here]

For this model the equation of motion of the jumper's centre of mass in the upward propulsion phase is

$$
F_{\text {bottom }}\left[1-\left(\frac{X-X_{\text {bottom }}}{X_{\text {take-off }}-X_{\text {bottom }}}\right)^{A}\right]-m g=m \frac{d^{2} X}{d t^{2}}
$$

where $m$ is the mass of the jumper, $g$ is the acceleration due to gravity $\left(9.812 \mathrm{~m} / \mathrm{s}^{2}\right.$ in our laboratory), and $d^{2} X / d t^{2}$ is the second (acceleration) derivative of position $(X)$ with respect to time $(t)$. The equation of motion is non-linear and so a technical computing software package (Mathematica v11.3; Wolfram Research, Champaign, IL, USA) was used to calculate the jump trajectory using numerical methods. In the simulated jumps the body weight, depth of countermovement, force at the bottom of the countermovement, and height at take-off were set to the mean value of the experimental jumps by the participant. The exponent was set so as to produce a jump height equal to the mean value of the experimental jumps by the participant.

One thousand (1000) jumps were simulated with small random changes in $X$ bottom, $F_{\text {bottom, and }} A$. The random changes in the three variables were independent of each other and were sampled from a normal distribution with a standard deviation equal 
to that of the experimental jumps by the participant. (The standard deviation in $A$ was set to produce changes in jump height similar to those produced by the changes in $X_{\text {bottom }}$ and $\left.F_{\text {bottom. }}\right)$ For each of the simulated jumps the same variables as in the experimental jumps were recorded. As with the experimental jumps, scattergraphs of pairs of variables were created and the corresponding Pearson's correlation coefficients were calculated.

The simulated jump data were intended to be compared to the experimental jump data from the participant. Further sets of simulated jump data were generated to represent jump data expected from other athletes. Sets of 1000 jumps were simulated with systematic changes in $X_{\text {bottom, }} F_{\text {bottom, and }} A$ over the range expected to be seen in physically active adult participants $\left(X_{\text {bottom }}=0.20,0.35\right.$, and $0.50 \mathrm{~m} ; F_{\text {bottom }}=1.5,2.5$, and $3.5 \mathrm{mg} ; A=3.0,4.5$, and 6.0).

\section{Results}

Each of the three participants produced a consistent set of experimental jumps with small variability in the force-time and force-position curves and with small variability in the jump variables (Table 2 and Supplemental Online Material 2). Data for all the jump variables were consistent with sampling from a normal distribution.

[Table 2 near here]

Jump height was strongly or very strongly correlated with peak power (Table 3; Figure 4; Supplemental Online Material 2), and the observed correlation was within 0.003 of the value calculated from the statistical theory for when the outcome variable is the product of two other variables (equation A7). The correlation between jump height and the velocity at peak power was very strong or near-perfect. The correlation between jump height and peak power was largely determined by the correlation between jump height and the velocity at peak power, and was not related to the correlation between 
jump height and the ground reaction force at peak power or to the correlation between the ground reaction force at peak power and the velocity at peak power. Jump height was also strongly or very strongly correlated with average power, and peak power was strongly or very strongly correlated with average power.

[Table 3 near here]

[Figure 4 near here]

The force-time and force-position curves for the simulated jumps were similar to the experimental jumps produced by the participant (Supplemental Online Material 3). For each of the three participants, the qualitative findings for the simulated jump data were similar to those for the experimental jumps data (Figure 4; Supplemental Online Material 2 and 3). Systematic changes in the values of $X_{\text {bottom, }} F_{\text {bottom, and } A \text { over }}$ a wide range did not affect the qualitative findings from the simulated jump data (Supplemental Online Material 3).

\section{Discussion and Implications}

The results indicate we should accept the initial hypothesis. In a random set of countermovement jumps by a participant the peak power and average power were strongly correlated with jump height. The observed correlation between jump height and peak power was in very close agreement with the value calculated from the statistical theory, and the correlation between jump height and the velocity at peak power was near-perfect. The correlation between jump height and peak power was largely determined by the correlation between jump height and the velocity at peak power, and was not related to the correlation between jump height and the ground reaction force at peak power or to the correlation between the ground reaction force at peak power and the velocity at peak power. These results support the argument that the mechanism for the strong correlation between jump height and peak power is that power 
is a compound variable calculated from the product of instantaneous ground reaction force and instantaneous velocity, with the correlation between jump height and power being artificially inflated by the near-perfect correlation between jump height and the velocity at peak power. The results from the simulated jump data indicate that the results from the experimental jump data are not specific to the participants in the study; there are physical mechanisms that underlie the observed correlations in the participants and so similar findings can be expected in other athletes.

\section{Critical assessment}

The present study used a within-subject correlation analysis of jump variables by a participant. The experimental jump data was a large set of maximal-effort jumps by the participant. Any differences among the jumps were assumed to be random inter-trial variations. That is, the experimental jump data were similar to that which would be collected in a baseline assessment of an athlete. Caution is required when interpreting the observed correlations in the experimental jump data. Although there were some strong correlations between variables, these correlations do not necessarily indicate causal mechanisms (Hay, 1985; Motulsky, 2018). A countermovement jump has complex interactions between the variables, and some important variables might not have been recorded in the present study. The correlations observed in the present study are likely to be just a glimpse of a more complicated set of relationships. An interpretation of the correlations between variables in a countermovement needs to be guided by a theoretical model of the mechanical factors that determine the jump height.

The mathematical model used to generate the simulated jump data was chosen to be as simple as possible while containing the essential features with the fewest number of adjustable parameters. It was assumed that the ground reaction force at any given position is primarily determined by the mechanical leverage of the body segments. The 
model did not include the force-velocity effects of muscle and the elastic energy effects of muscle and tendon, and so any time-dependent changes in the ground reaction forceposition curve were not considered. Also, the force-position profile in the model was represented by a monotonically decreasing curve (a power equation) and so any 'bimodal' effects in the force curve were ignored (Kennedy \& Drake, 2018). Despite these omissions, the model produced force-time and force-position curves that were broadly similar to the experimental jumps produced by the participants.

Even though the model was intended to apply to inter-trial variations in an individual athlete, the strength and direction of the correlations in the simulated jump data did not always agree with those observed in the experimental jump data. Discrepancies could be due to incorrect or missing causal relationships in the model. For example, experimental intervention studies have shown that a greater countermovement depth reduces the force at the start of the upward propulsion phase and has a small effect on the jump height (Linthorne, 2000; Mandic, Jakovljevic, \& Jaric, 2015). Likewise, a greater rate of countermovement produces a greater force at the bottom of the countermovement and a greater jump height (Linthorne, 2001). These causal relationships were not always evident in the correlation plots from the participants in the present study (Supplemental Online Material 2), However, causal relationships are not always evident in a within-subject correlation study when the intertrial variations are relatively small (Hay, 1985).

The simulated jump data produced correlations that were stronger than in the experimental jump data. This could be because the simple model does not include all the complexity and variability of a real countermovement jump. When interpreting the data from the simulated jumps, attention was paid to the relative differences in the strengths of the correlations rather than the absolute values. The statistical theory 
predicts that the correlation between jump height and peak power will be largely determined by the correlation between jump height and the velocity at peak power and will not necessarily be related to the correlation between jump height and the force at peak power.

The present study did not use the model of jumping proposed by Samozino, Morin, Hintzy, and Belli (2008) because this model only considers the average power during the upward propulsion phase of the jump; instantaneous power is not considered. Also, for the participants in the present study the equations proposed by Samozino et al for the average force, average velocity, and average power in the upward propulsion phase of the jump give values that are about $10-25 \%$ less than the time-average values obtained from the force platform (Supplemental Online Material 4). The discrepancy could be due to Samozino et al's lack of distinction between time-average and positionaverage quantities, which are not numerically the same. In the upward propulsion phase of a countermovement jump the difference between the time-average quantity and the position-average quantity is about $10 \%$ for ground reaction force, about $30 \%$ for velocity, and about 20\% for power (Supplemental Online Material 4). Samozino et al also used an approximate method for calculating the vertical push-off distance, and for calculating the jump height they used the flight-time method which gives a slightly greater value than the more accurate impulse-momentum method (Supplemental Online Material 4).

\section{Comparison with other studies}

When assessing athletes using a performance test, the trainer usually needs to make a decision about the individual athlete rather than about a group of athletes or a whole team (McGuigan, 2017; Sands et al., 2019). Therefore, the present study used a withinsubject correlation analysis. However, all previous correlation studies used a between- 
subject analysis in which one jump by a large number of participants was analysed; I did not identify any previous within-subject studies that analysed a substantial number of jumps by a single participant. The within-subject analysis in the present study revealed a strong or very strong correlation between jump height and peak power $(0.54-$ 0.83 ) and between jump height and average power (0.63-0.88). Similar correlations have been seen in between-subject studies; $0.80-0.93$ for the correlation between jump height and peak power (Aragón-Vargas \& Gross, 1997; Barker et al., 2017; Dowling \&Vamos, 1993; Harman et al., 1990; Markovic et al., 2014), and 0.65 for the correlation between jump height and average power (Markovic et al., 2014).

Caution is required when comparing results from studies because a betweensubject study and a within-subject study will not always identify the same variables as being strongly correlated with performance (Hay, 1985). If a variable truly has a causal influence on jump height and the participant is highly consistent in that variable, then a within-subject correlation study will not produce a strong correlation with jump height. Likewise, in a between-subject study an important causal variable might produce only a low correlation with jump height if there are substantial differences among the participants in another causal variable.

\section{Limitations of the study}

This study used a single-subject analysis with three participants; however, the low number of participants is not a substantial limitation. There have been misconceptions about the advantages and limitations of the single-subject approach (Bates, 1996; Bates et al., 2004). Some have questioned the statistical power of this type of study and whether the low number of participants limits the generalisability of the findings. A single-subject analysis involves a detailed testing of a single participant using many trials and can produce strong evidence in support or against a particular hypothesis as 
applied to that participant. The statistical power that is relevant here is mainly determined by the number of trials for that participant; not by the number of participants. The generalisability of the findings is addressed through replication (i.e., testing the hypothesis on additional participants). For a study in which there are three participants who have been randomly chosen from a population, the probability that all three participants produce the same result is relatively small (less than 5\%) (Bates et al., 2004). Therefore, the findings from the present study can be expected to be representative of healthy young adult male and females who are physically active and compete regularly in sports that involve running and jumping.

However, the findings from the present study might not apply to other populations and other types of jump. The argument presented in the Introduction about the correlation between jump height and peak power assumes the jumper has force, velocity, and power curves similar to those shown in Figures 1 and 2. In particular, the jumper is assumed to have a near-linear relation between velocity and position during the upward propulsion phase. For a person who does not satisfy these assumptions, the findings from the present study might not apply. Potential persons include children, older adults, persons with limited experience of performing a countermovement jump, amputees with prosthetics, persons with substantial neuromuscular disease or neuromuscular injury, and persons with substantial inter-limb differences in strength, length, or coordination. The findings from the present study probably apply to healthy young adult athletes when performing a squat jump. A squat jump usually has a nearlinear relation between velocity and position during the upward propulsion phase and so the take-off velocity should be very strongly correlated with the velocity at any given position. However, the findings from the present study might not apply to a drop jump, 
a set of rebound jumps, or a heavy loaded jump squat if these types of jump do not have a near-linear relation between velocity and position.

\section{Implications for the assessment of athletes}

The aim of the present study was to explain the underlying cause of the strong correlation between jump height and mechanical power in a countermovement jump. The finding that the correlation is artificially inflated is not, by it itself, an argument against using mechanical power to assess the neuromuscular performance of an athlete. A countermovement jump is widely believed to give information about changes in an athlete's neuromuscular function and stretch-shorten cycle function (Claudino et al., 2017). Coaches and trainers use the countermovement jump to monitor the short-term changes in response to a single training session or a competition, and to monitor the longer-term changes in response to a block of training or a block of competitions. The relevant aspects of neuromuscular function and stretch-shorten cycle function that can change include the force-generating capacity of the muscle (both central and peripheral) and the athlete's stretch-shorten cycle capability (as indicated by the coupling of the downward and upward phases in a countermovement jump).

\section{Average power}

Some investigators have suggested that average mechanical power might be a performance-limiting factor in a countermovement jump (Bobbert, 2014; Lake \& Mundy, 2018). In a countermovement jump there is a cause-and-effect relation between the resultant work performed on the jumper's centre of mass during the upward propulsion phase ( $\left.W_{\text {Res }}\right)$ and jump height. The resultant force on the athlete is given by $F_{\mathrm{Res}}=F_{\mathrm{GRF}}-m g$, and so there is also a cause-and-effect relation between the work performed by the ground reaction force during the upward propulsion phase ( $\left.W_{\mathrm{GRF}}\right)$ and 
jump height. In a countermovement jump the jump height will increase if the jumper performs more work (by, for example, exerting a greater ground reaction force over the same vertical range of motion). The time-average mechanical power flow to the ground in the upward propulsion phase of the jump is given by $P_{\mathrm{GRFav}}=\mathrm{W}_{\mathrm{GRF}} / \Delta t$, where $\Delta t$ is the duration of the upward propulsion phase. However, $W_{\mathrm{GRF}}$ and $\Delta t$ are inter-related. By performing more work, the jumper's centre of mass has a greater upward acceleration and so moves through the vertical propulsion distance in less time (i.e., the duration is less). The increase in work and the decrease in duration both act to increase the average mechanical power. This suggests that average mechanical power might be a performance-limiting factor in a time-constrained action such as a countermovement jump (Bobbert, 2014; Lake \& Mundy, 2018). That is, to be able to jump higher the athlete must be able to produce a greater average power flow to the ground during the upward propulsion phase.

However, the above argument assumes that the range of motion (i.e., the depth of countermovement) is constant and that the jumper always performs the downward phase of the jump in the same way. Experimental intervention studies on skilled jumpers have shown that jump height increases slightly with increasing countermovement depth, and that jump height increases with increasing rate of countermovement (due to an increase the force at the bottom of the countermovement) (Linthorne 2000; Linthorne, 2001). These studies have also shown that average power increases with increasing countermovement depth and increases with increasing rate of countermovement. The confounding effects of countermovement depth and the rate of countermovement mean that the relation between jump height and average mechanical power in a countermovement jump is not unique; there are many combinations of countermovement depth and rate of countermovement that produce the same jump 
height, and these jumps can have substantially different values of average mechanical power. Producing a greater average power does not always lead to a greater jump height. Although average mechanical power is strongly linked to jump height, average mechanical power should not be considered as a performance-limiting factor in a countermovement jump.

Average mechanical power could still be useful in monitoring and assessing athletes. A change in average mechanical power during the upward propulsion phase of the jump might indicate that the force generating capability of the athlete's muscles has changed (e.g., the average ground reaction force has changed), or that some aspect of the athlete's jumping technique (e.g., countermovement depth, rate of countermovement) has changed. Although average mechanical power appears to be a sensitive indicator of changes in neuromuscular function and stretch-shorten cycle function (Claudino et al., 2017), it does not directly reveal the reason(s) for the change. To interpret a change in average mechanical power, other variables might also need to be examined (Nimphius, 2017).

\section{Peak power}

The relation between jump height and peak mechanical power is also unlikely to be unique; the relationship is likely to be confounded by countermovement depth and the rate of countermovement. The relationship might also be confounded by the shape of the force-position curve. The jump height achieved in a countermovement jump depends on the shape of the force-position curve in the upward propulsion phase

(Figure 2). For a given countermovement depth and rate of countermovement, an athlete who is able to 'square the pulse' will perform more work (i.e., the area under the curve will be greater) and so will have a greater jump height (Adamson \& Whitney, 1971). A sharper decrease in ground reaction force toward the end of the upward propulsion 
phase could arise from a more optimal timing of the proximal-distal extension of the hip, knee, and ankle joints. In the simple countermovement jump model used in the present study (Figure 3; Equation 2), a sharper decrease in ground reaction force is equivalent to a greater power exponent, $A$. A greater $A$ results in a greater peak power and a higher position of the jumper's centre of mass at peak power. Therefore, a higher position at peak power might be an indicator of a superior jumping technique where the athlete has greater ability to produce force toward the end of the take-off.

For the three participants in the present study there was a small to strong positive correlation between jump height and the position at peak power (about 0.1$0.4 \pm 0.2$ ) (Supplemental Online Material 2). However, the uncertainty in the measurement of the position of the participant's centre of mass at peak power is relatively large (about $0.010 \mathrm{~m}$ ) and so the observed correlations might not be accurate. Also, the uncertainty is comparable to the participant's inter-trial variability, and this substantially reduces our ability to detect a meaningful change in the position at peak power when monitoring jumps by an athlete (Hopkins, 2004).

Modelling studies have indicated that the ability to produce a high peak power might be important in avoiding a premature take-off where the feet leave the ground before the hip, knee, and ankle joints reach full extension (Bobbert \& Van Soest, 2001). To reach full extension, the model results indicate that instantaneous mechanical power flow to the ground must continue to increase during the upward propulsion phase. Therefore, some researchers have suggested that peak mechanical power might be a performance-limiting factor in a countermovement jump (Bobbert, 2014; Lake \& Mundy, 2018). However, none of the three participants in the present study showed a strong correlation between take-off height and peak power (Supplemental Online Material 2). This finding could be interpreted as evidence that the ability to produce a 
high peak power is not a limiting factor in a countermovement jump, but an experimental study in which peak power is deliberately manipulated is more likely to resolve the issue.

Jump height and peak power are currently the most common variables used in the assessment of neuromuscular capacity in elite athletes (Owen, Watkins, Kilduff, Bevan, \& Bennet, 2014). However, the peak power obtained from a force platform is not a direct measure of the physiological power generated by the athlete's muscles. Unfortunately, the terms 'power' and 'peak' might be subconsciously attractive to some coaches and sport scientists. A measurement of peak power from a force platform is a very close (but not exact) indicator of an athlete's jumping ability. Peak power should not be seen as a new 'performance variable' that is substantially different from jump height. The biomechanical link between jump height and peak power is illustrated in Figure 5 (and Supplemental Online Material 2).

[Figure 5 near here]

In summary, mechanical power from a force platform appears to be a sensitive variable for identifying when 'something has changed' in the athlete's physical capacity or in the athlete's jumping technique. However, an increase in mechanical power in a jump does not necessarily represent an improvement in neuromuscular capacity or stretch-shorten cycle function, and likewise a decrease does not necessarily represent a deterioration. To interpret a change in mechanical power, other variables such as countermovement depth, rate of countermovement, and the timing of joint extensions might need to be investigated.

\section{Conclusion}

In a set of countermovement jumps by an athlete, the mechanical power in the upward propulsion phase is strongly correlated with jump height. However, this strong 
correlation is an artefact arising from the fact that power is a compound variable calculated from the product of instantaneous ground reaction force and instantaneous velocity. The correlation between jump height and peak power is artificially inflated by the near-perfect correlation between jump height and the velocity at peak power.

\section{Acknowledgements}

Thanks to Antonia Gregoriou and Husan Thapa for providing the experimental jump data.

\section{Disclosure Statement}

No potential conflict of interest was reported by the author.

\section{References}

Adamson, G. T., \& Whitney, R. J. (1971). Critical appraisal of jumping as a measure of human power. In J. Vredenbregt, \& J. Wartenweiler (Eds.), Medicine and Sport 6, Biomechanics II (pp. 208-211). Basel, Switzerland: S. Karger.

Aragón-Vargas, L. F. \& Gross, M. M. (1997). Kinesiological factors in vertical jump performance: Differences among individuals. Journal of Applied Biomechanics, 13, 24-44. doi: 10.1249/00005768-199505001-00956

Barker, L. A., Hart, J. R., \& Mercer, J. A. (2017). Relationships between countermovement jump ground reaction forces and jump height, reactive strength index, and jump time. Journal of Strength and Conditioning Research, 21, 248-254. doi: 10.1519/JSC.0000000000002160

Bates, B. T. (1996). Single-subject methodology: An alternative approach. Medicine \& Science in Sports \& Exercise, 28, 631-638. doi: 10.1097/00005768199605000-00016 
Bates, B. T., James, C. R., \& Dufek, J. S. (2004). Single-subject analysis. In N. Stergiou (Ed.), Innovative analyses of human movement (pp. 3-28). Champaign, IL: Human Kinetics.

Bobbert, M. F. (2014). Effect of unloading and loading on power in simulated countermovement and squat jumps. Medicine \& Science in Sports \& Exercise, 46, 1176-1184. doi: 10.1249/MSS.0000000000000216

Bobbert, M. F., \& Van Soest, A. J. (2001). Why do people jump the way they do? Exercise and Sport Sciences Reviews, 29, 95-102. doi: 10.1097/00003677200107000-00002

Bohrnstedt, G. W., \& Goldberger, A. S. (1969). On the exact covariance of products of random variables. Journal of the American Statistical Association, 64, 1439-1442. doi: 10.1080/01621459.1969.10501069

Claudino, J. G., Cronin, J., Mezêncio, B., McMaster, D. T., McGuigan, M., Tricoli, V., Amadio, A. C., \& Serrão, J. C. (2017). The countermovement jump to monitor neuromuscular status: A meta-analysis. Journal of Science and Medicine in Sport, 20, 397-402. doi: 10.1016/j.jsams.2016.08.011

Dowling, J. J., \& Vamos, L. (1993). Identification of kinetic and temporal factors related to vertical jump performance. Journal of Applied Biomechanics, 9, 95110. doi: $10.1123 /$ jab.9.2.95

Goodman, L. A. (1960). On the exact variance of products. Journal of the American Statistical Association, 55, 708-713. doi: 10.1080/01621459.1960.10483369

Knudson, D. V. (2009). Correct use of the term "power" in the strength and conditioning literature. Journal of Strength and Conditioning Research, 23, 1902-1908. doi: 10.1519/JSC.0b013e3181b7f5e5 
Harman, E. A., Rosenstein, M. T., Frykman, P. N., \& Rosenstein, R. M. (1990). The effects of arms and countermovement on vertical jumping. Medicine \& Science in Sports \& Exercise, 22, 825-833. doi: 10.1249/00005768-199012000-00015

Hay, J. G. (1985). Issues in sports biomechanics. In S. M. Perren, \& E. Schneider (Eds.), Biomechanics: Current interdisciplinary research (pp. 49-60).

Dordrecht, The Netherlands: Martinus Nijhoff Publishers.

Hopkins, W. (2004). How to interpret changes in an athletic performance test. Sportscience, 8, 1-7.

Hopkins, W. G., Marshall, S. W., Batterham, A. M., \& Hanin, J. (2009). Progressive statistics for studies in sports medicine and exercise science. Medicine \& Science in Sports \& Exercise, 41, 3-13. doi: 10.1249/MSS.0b013e31818cb278

Kennedy, R. A., \& Drake, D. (2018). Is a bimodal force-time curve related to countermovement jump performance? Sports, 6, 36. doi: 10.3390/sports6020036

Lake, J. P., \& Mundy, P. (2018). Assessment of power. In P. Comfort, P. A. Jones, \& J. J. McMahon (Eds.), Performance assessment in strength and conditioning (pp. 193-211). London, England: Routledge.

Linthorne, N.P. (2000). Optimum take-off range in vertical jumping. In R. Barrett, R. Simeoni, \& C. D'Helon (Eds.), Book of Abstracts, 3rd Australasian Biomechanics Conference, Griffith University, Gold Coast, Australia, 31 January - 1 February 2000 (pp. 49-50). Gold Coast, Australia: Griffith University, School of Physiotherapy and Exercise Science.

Linthorne, N. P. (2001). Analysis of standing vertical jumps using a force platform. American Journal of Physics, 69, 1198-1204. doi: 10.1119/1.1397460 
Mandic, R., Jakovljevic, S., \& Jaric, S. (2015). Effects of countermovement depth on kinematic and kinetic patterns of maximum vertical jumps. Journal of Electromyography and Kinesiology, 25, 265-272. doi: 10.1016/j.jelekin.2014.11.001

Markovic, S., Mirkov, D. M., Nedeljkovic, A., \& Jaric, S. (2014). Body size and countermovement depth confound relationship between muscle power output and performance. Human Movement Science, 33, 203-210. doi:

10.1016/j.humov.2013.11.00

McGuigan, M. (2017). Monitoring training and performance in athletes. Champaign, IL: Human Kinetics.

McMahon, J. J., Lake, J. P., \& Suchomel, T. J. (2018). Vertical jump testing. In P. Comfort, P. A. Jones, \& J. J. McMahon (Eds.), Performance assessment in strength and conditioning (pp. 96-116). London, England: Routledge.

Morin, J.-B., Jiménez-Reyes, P., Brughelli, M., \& Samozino, P. (2019). When jump height is not a good indicator of lower limb maximal power output: Theoretical demonstration, experimental evidence and practical solutions. Sports Medicine, 49, 999-1006. doi.org/10.1007/s40279-019-01073-1

Motulsky, H. (2018). Intuitive biostatistics (4th ed.). Oxford, England: Oxford University Press.

Nimphius, S. (2017). Assessment of power. In M. McGuigan (Ed.). Developing power (pp. 15-32). Champaign, IL: Human Kinetics.

Owen, N. J., Watkins, J., Kilduff, L. P., Bevan, H. R., Bennet, M. A. (2014). Development of a criterion method to determine peak mechanical power output in a countermovement jump. Journal of Strength and Conditioning Research, 28, 15521558. doi: $10.1519 / \mathrm{JSC} .0000000000000311$ 
Sands, W., Cardinale, M., McNeal, J., Murray, S., Sole, C., Reed, J., Apostolopoulos, N, Stone, M. (2019). Recommendations for measurement and management of elite athletes. Sports, 7, 105. doi: 10.3390/sports7050105

Samozino, P., Morin, J.-B., Hintzy, F., \& Belli, A. (2008). A simple method for measuring force, velocity, and power output during squat jump. Journal of Biomechanics, 41, 2940-2945. doi: 10.1016/j.jbiomech.2008.07.028

Winter, E. M. (2005). Jumping: Power or impulse? Medicine \& Science in Sports \& Exercise, 37, 523. doi: 10.1007/s004210050673

\section{Appendix: Correlation Coefficient when the Output Variable is the Product of Two Other Variables}

Consider three random variables $X, Y$, and $Z$, with mean values $\mu_{X}, \mu_{Y}$, and $\mu_{Z}$, and standard deviations $\sigma_{X}, \sigma_{Y}$, and $\sigma_{Z}$. The correlation coefficient for two variables (say, $X$ and $Y$ ) is defined as

$$
\rho_{X, Y}=\frac{\operatorname{cov}(X, Y)}{\sigma_{X} \sigma_{Y}}
$$

where $\operatorname{cov}(X, Y)$ is the covariance of $X$ and $Y$.

Now consider a new variable created as the product of $Y$ and $Z$. The correlation between $\mathrm{X}$ and $Y Z$ is given by

$$
\rho_{X, Y Z}=\frac{\operatorname{cov}(X, Y Z)}{\sigma_{X} \sigma_{Y Z}}
$$

where $\sigma_{Y Z}$ is the standard deviation of $Y Z$, and $\operatorname{cov}(X, Y Z)$ is the covariance of $X$ and $Y Z$. If $X, Y$, and $Z$ are multivariate normal, the covariance of $X$ and $Y Z$ is given by (Bohrnstedt \& Goldberger, 1969) 


$$
\operatorname{cov}(X, Y Z)=\mu_{Y} \operatorname{cov}(X, Z)+\mu_{Z} \operatorname{cov}(X, Y)
$$

where $\operatorname{cov}(X, Y)$ is the covariance of $X$ and $Y$, and $\operatorname{cov}(X, Z)$ is the covariance of $X$ and $Z$. Also, the standard deviation of $Y Z$ is given by (Bohrnstedt \& Goldberger, 1969;

Goodman, 1960)

$$
{\sigma_{Y Z}}^{2}=\mu_{Y}^{2}{\sigma_{Z}}^{2}+\mu_{Z}^{2}{\sigma_{Y}}^{2}+{\sigma_{Y}}^{2}{\sigma_{Z}}^{2}+2 \mu_{Y} \mu_{Z} \operatorname{cov}(Y, Z)+(\operatorname{cov}(Y, Z))^{2}(\mathrm{~A} 4)
$$

Substituting equations A3 and A4 into equation A2 gives

$$
\rho_{X, Y Z}=\frac{\mu_{Y} \operatorname{cov}(X, Z)+\mu_{Z} \operatorname{cov}(X, Y)}{\sigma_{X} \sqrt{\mu_{Y}^{2} \sigma_{Z}^{2}+\mu_{Z}^{2} \sigma_{Y}^{2}+\sigma_{Y}^{2} \sigma_{Z}^{2}+2 \mu_{Y} \mu_{Z} \operatorname{cov}(Y, Z)+(\operatorname{cov}(Y, Z))^{2}}}
$$

Equation A5 can be re-written in terms of the correlations between $X, Y$, and $Z$;

$$
\rho_{X, Y Z}=\frac{\mu_{Y} \sigma_{Z} \rho_{X, Z}+\mu_{Z} \sigma_{Y} \rho_{X, Y}}{\sqrt{\mu_{Y}^{2} \sigma_{Z}^{2}+\mu_{Z}^{2} \sigma_{Y}^{2}+\sigma_{Y}^{2} \sigma_{Z}^{2}+2 \mu_{Y} \mu_{Z} \sigma_{Y} \sigma_{Z} \rho_{Y, Z}+\left(\sigma_{Y} \sigma_{Z} \rho_{Y, Z}\right)^{2}}}
$$

where $\rho_{X, Y}$ is the correlation between $X$ and $Y, \rho_{X, Z}$ is the correlation between $X$ and $Z$, and $\rho_{Y, Z}$ is the correlation between $Y$ and $Z$.

In a countermovement jump with a jump height, $H$, the peak power during the upward propulsion phase $(P)$ is calculated as the product of the ground reaction force at peak power $(F)$ and the velocity at peak power $(V)$ (i.e., $P=F V)$. The correlation between jump height and the peak power $\left(\rho_{H, P}\right)$ is then given by

$$
\rho_{H, P}=\frac{\mu_{F} \sigma_{V} \rho_{H, V}+\mu_{V} \sigma_{F} \rho_{H, F}}{\sqrt{\mu_{F}^{2} \sigma_{V}^{2}+\mu_{V}^{2} \sigma_{F}^{2}+\sigma_{F}^{2} \sigma_{V}^{2}+2 \mu_{F} \mu_{V} \sigma_{F} \sigma_{V} \rho_{F, V}+\left(\sigma_{F} \sigma_{V} \rho_{F, V}\right)^{2}}}
$$

where $\rho_{H, F}$ is the correlation between jump height and the force at peak power; $\rho_{H, V}$ is the correlation between jump height and the velocity at peak power; $\rho_{F, V}$ is the correlation between the force at peak power and the velocity at peak power; $\sigma_{H}, \sigma_{F}$, and $\sigma_{V}$ are the standard deviations of $H, F$, and $V$; and $\mu_{H}, \mu_{F}$, and $\mu_{V}$ are the mean values of 
$H, F$, and $V$.

In a countermovement jump by a skilled athlete, $\sigma_{F} / \mu_{F}$ and $\sigma_{V} / \mu_{V}$ (i.e., the ratio of the standard deviation to the mean value) are usually less than about 4\% (Supplemental Online Material 2). Therefore, the third and fifth terms in the denominator of equation A7 are negligible in comparison to the first, second, and fourth terms, even if the correlation between the force at peak power and the velocity at peak power $\left(\rho_{F, V}\right)$ is close to \pm 1 . Equation A7 can then be written as

$$
\rho_{H, P} \approx \frac{a}{\sqrt{a^{2}+b^{2}+c\left(\rho_{F, V}\right)}} \rho_{H, V}+\frac{b}{\sqrt{a^{2}+b^{2}+c\left(\rho_{F, V}\right)}} \rho_{H, F}
$$

where $a=\mu_{F} \sigma_{V}, b=\mu_{V} \sigma_{F}$, and $c=2 \mu_{F} \mu_{V} \sigma_{F} \sigma_{V}$. That is, $\rho_{H, P}$ is determined by a sum of $\rho_{H, V}$ and $\rho_{H, F}$, with scale factors that include a small effect from $\rho_{F, V}$. Most skilled athletes can expect to have scale factors of between about 0.6 and 1.2. For the three participants in the present study, the expected correlations between jump height and peak power calculated using equation A7 are 0.83, 0.64, and 0.55 (Supplemental Online Material 5). 
Table 1. Participant characteristics.

\begin{tabular}{lccc}
\hline Characteristic & Participant 1 & Participant 2 & Participant 3 \\
\hline Sex & female & male & male \\
Age (years) & 23 & 22 & 20 \\
Height (m) & 1.70 & 1.71 & 1.80 \\
Body mass (kg) & 56 & 81 & 73 \\
\hline
\end{tabular}

Table 2. Descriptive statistics for the jump variables; mean \pm standard deviation.

\begin{tabular}{lccc}
\hline Variable & Participant 1 & Participant 2 & Participant 3 \\
\hline Jump height (m) & $0.274 \pm 0.016$ & $0.305 \pm 0.015$ & $0.327 \pm 0.018$ \\
Depth of countermovement (m) & $0.350 \pm 0.019$ & $0.337 \pm 0.026$ & $0.277 \pm 0.029$ \\
Force at bottom of the countermovement (N) & $1101 \pm 46$ & $1798 \pm 89$ & $1812 \pm 67$ \\
Maximum force during propulsion phase (N) & $1139 \pm 27$ & $1825 \pm 72$ & $1832 \pm 67$ \\
Take-off height (m) & $0.098 \pm 0.006$ & $0.108 \pm 0.010$ & $0.136 \pm 0.012$ \\
Duration of propulsion phase (s) & $0.320 \pm 0.011$ & $0.289 \pm 0.015$ & $0.254 \pm 0.017$ \\
Peak power (W) & $2484 \pm 85$ & $3895 \pm 127$ & $3877 \pm 130$ \\
Force at peak power (N) & $1090 \pm 27$ & $1642 \pm 53$ & $1601 \pm 60$ \\
Velocity at peak power (m/s) & $2.28 \pm 0.06$ & $2.37 \pm 0.05$ & $2.42 \pm 0.07$ \\
Position at peak power (m) & $-0.058 \pm 0.007$ & $-0.071 \pm 0.011$ & $-0.042 \pm 0.012$ \\
Average power (W) & $1258 \pm 51$ & $2099 \pm 79$ & $2130 \pm 90$ \\
Average force (N) & $972 \pm 19$ & $1510 \pm 40$ & $1477 \pm 50$ \\
Average velocity (m/s) & $1.40 \pm 0.05$ & $1.54 \pm 0.04$ & $1.62 \pm 0.04$ \\
& & & \\
\hline
\end{tabular}

Participant 1, 60 jumps; Participant 2, 56 jumps; Participant 3, 47 jumps 
Table 3. Strength of the correlation between jump variables; $r \pm 90 \%$ CI.

\begin{tabular}{lccc}
\hline Variables & Participant 1 & Participant 2 & Participant 3 \\
\hline Jump height - Peak power & $0.83 \pm 0.07$ & $0.63 \pm 0.13$ & $0.54 \pm 0.17$ \\
Jump height - Velocity at peak power & $0.94 \pm 0.03$ & $0.83 \pm 0.07$ & $0.90 \pm 0.05$ \\
Jump height - Force at peak power & $0.18 \pm 0.21$ & $0.08 \pm 0.22$ & $-0.20 \pm 0.23$ \\
Velocity at peak power - Force at peak power & $-0.05 \pm 0.21$ & $-0.32 \pm 0.20$ & $-0.51 \pm 0.18$ \\
Jump height - Average power & $0.88 \pm 0.07$ & $0.76 \pm 0.10$ & $0.63 \pm 0.15$ \\
Peak power - Average power & $0.75 \pm 0.10$ & $0.66 \pm 0.13$ & $0.90 \pm 0.05$
\end{tabular}

Participant 1, 60 jumps; Participant 2, 56 jumps; Participant 3, 47 jumps 


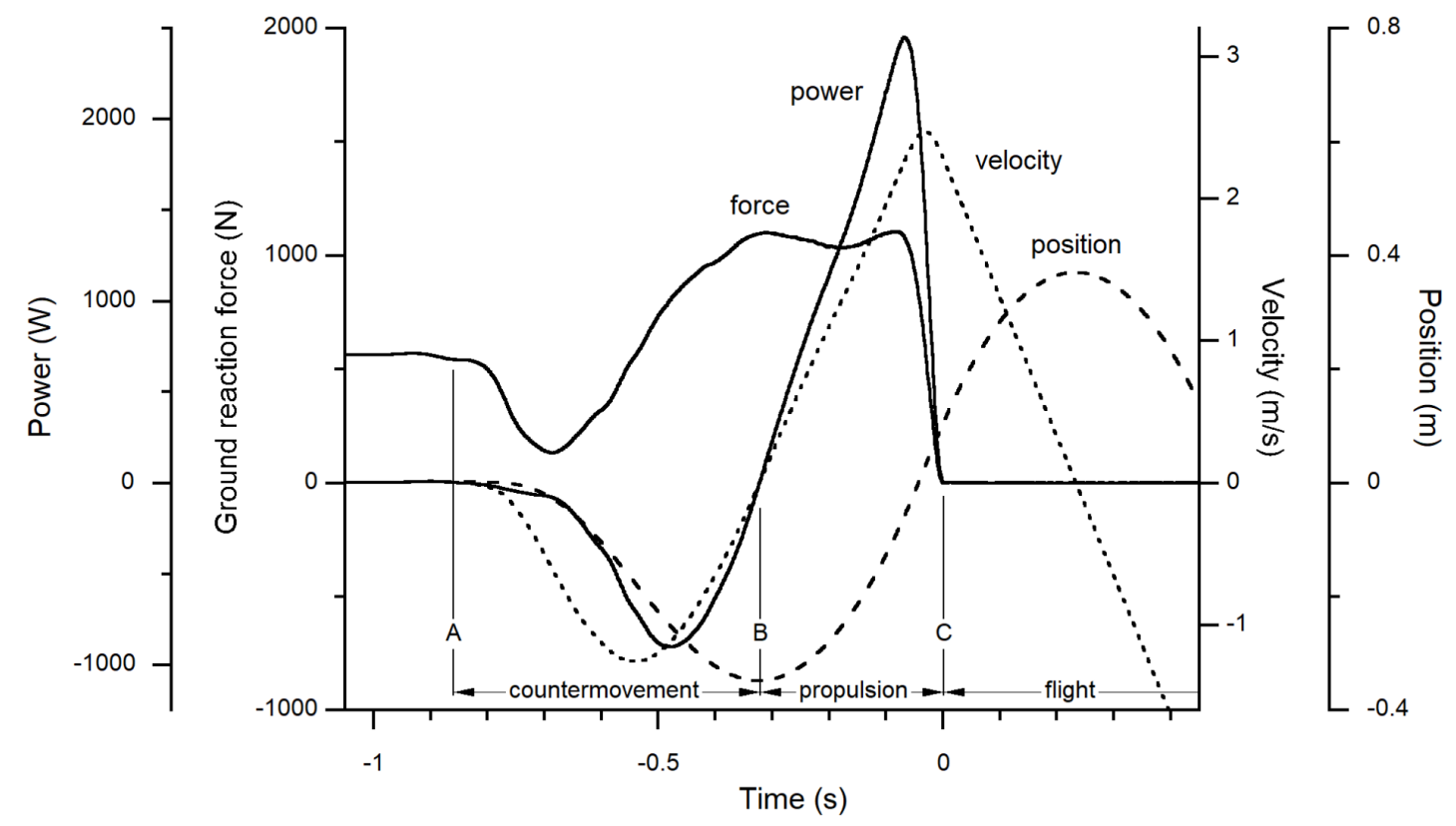

Figure 1. Time traces of position, velocity, ground reaction force, and power in a typical countermovement jump (without arm swing) by an experienced adult athlete. Velocity reaches a maximum shortly before take-off where the ground reaction force drops below body weight and the resultant force on the athlete becomes negative. Power is the product of the ground reaction force and velocity. Peak power occurs shortly before take-off, just as the ground reaction force begins to rapidly decrease. In this plot, time zero has been set to the instant of take-off. $\mathrm{A}=$ start; $\mathrm{B}=$ bottom; $\mathrm{C}=$ take-off 


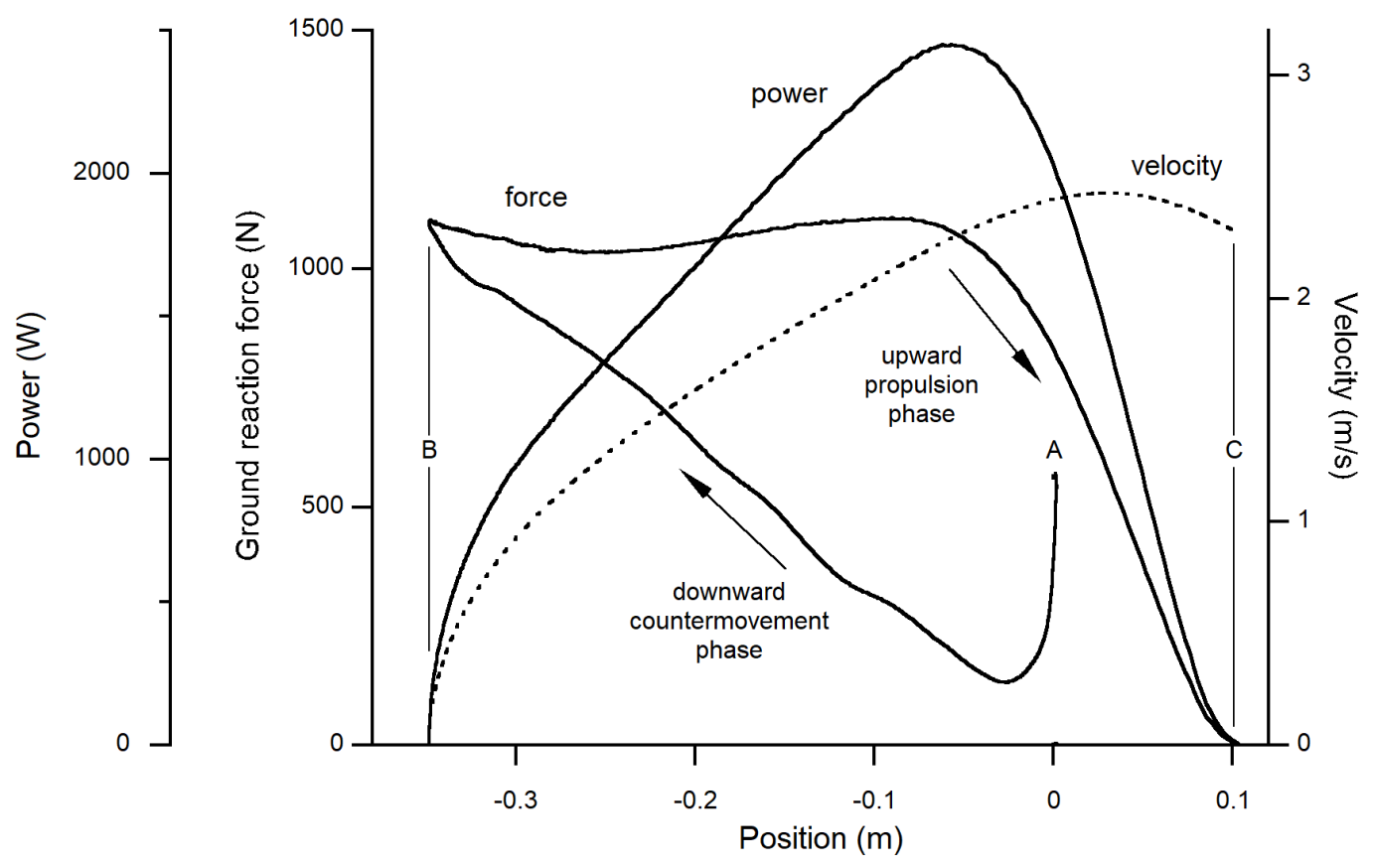

Figure 2. Position traces of velocity, ground reaction force, and power in a countermovement jump without arm swing. Data for the same jump as in Figure 1. For a substantial portion of the upward propulsion phase there is a near-linear relationship between velocity and position. Peak power usually occurs a few centimetres below the start position. The regions of negative velocity and power in the downward countermovement phase have been omitted for clarity. $\mathrm{A}=$ start; $\mathrm{B}=$ bottom; $\mathrm{C}=$ takeoff 


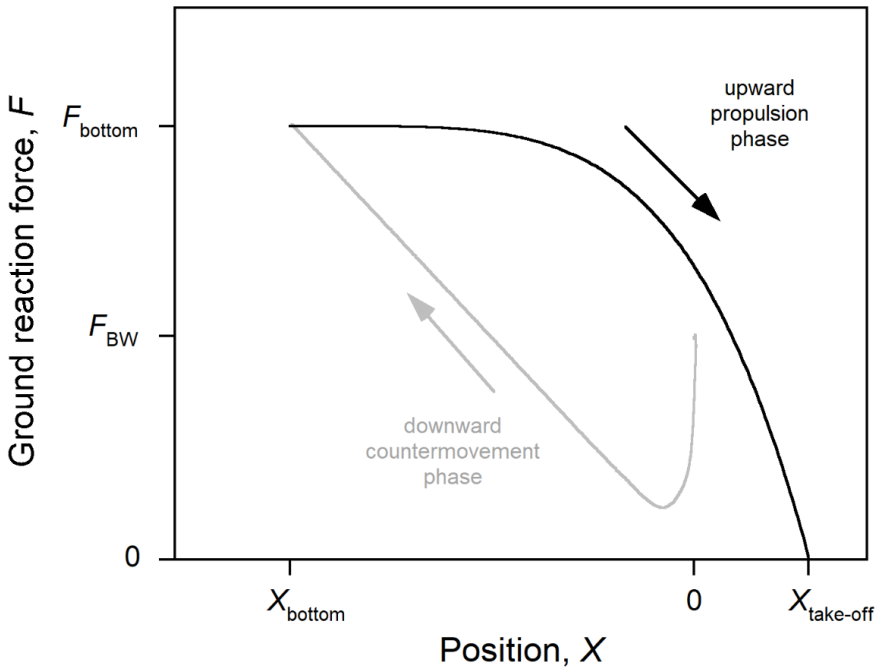

Figure 3. Model of a countermovement jump used to generate the simulated jump data. The jump height achieved by the jumper is determined by the work performed during the upward propulsion phase (i.e., the area under the force-position curve). The downward countermovement phase (grey curve) is not relevant to the model but is shown for completeness. Compare to Figure 2. 
Experimental Jumps
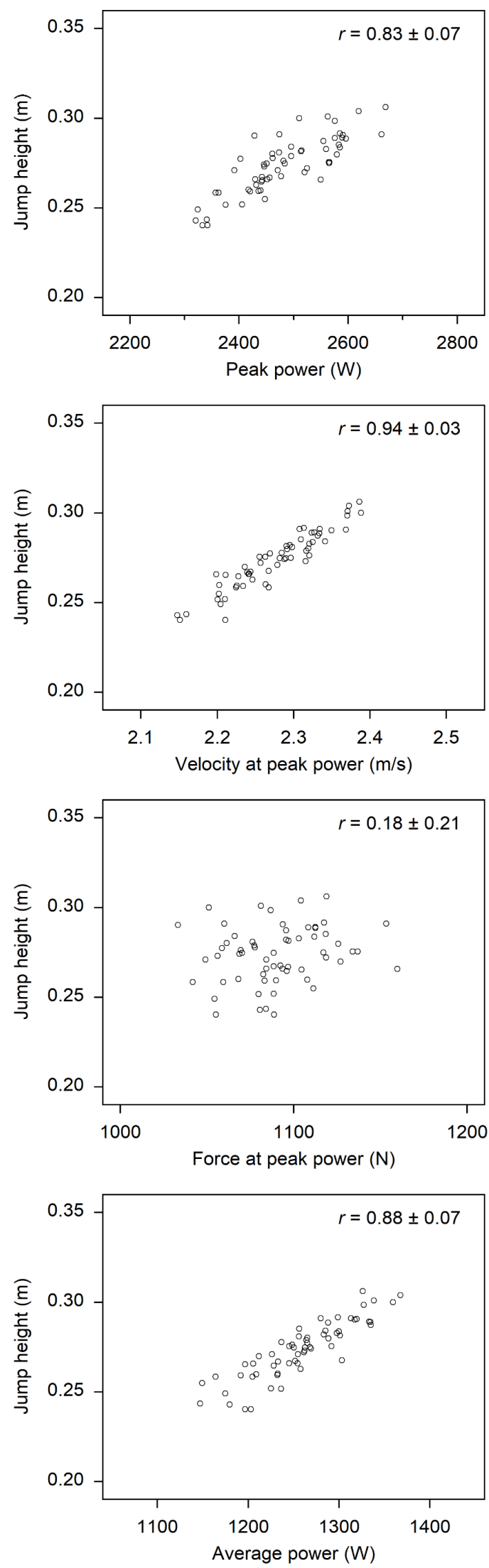

Simulated Jumps
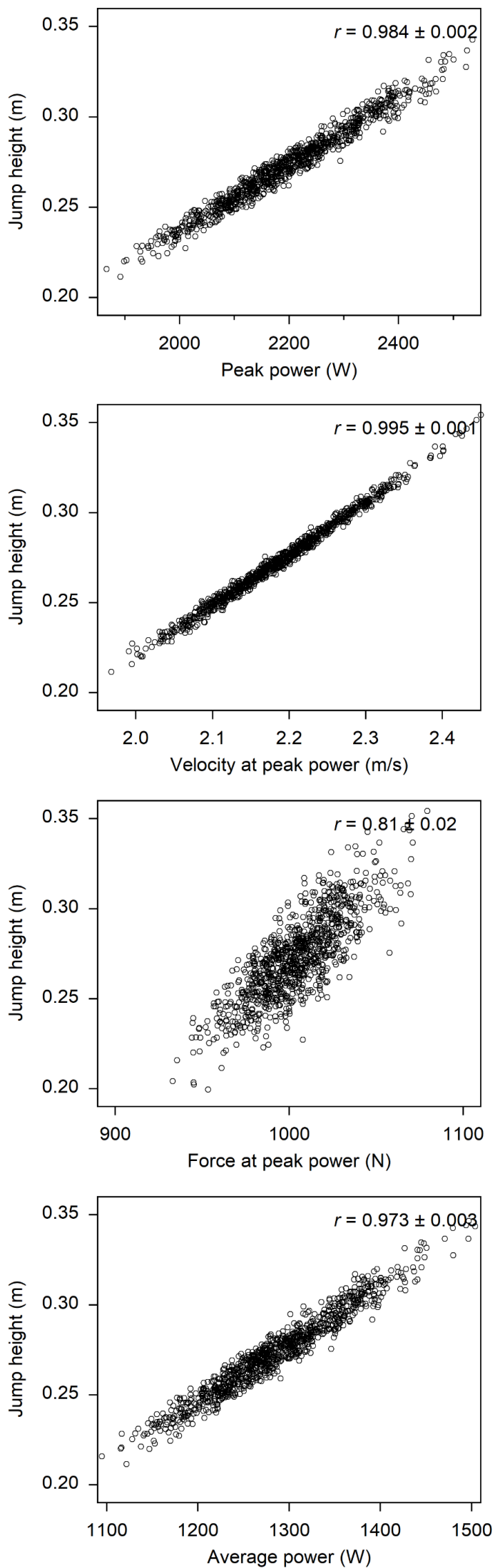
Figure 4. In the experimental jumps, jump height was strongly correlated with peak power $(r \pm 90 \% \mathrm{CI})$. The correlation between jump height and peak power was largely determined by the near-perfect correlation between jump height and the velocity at peak power, and was not related to the correlation between jump height and the force at peak power. Jump height was also strongly correlated with average power. Data for Participant 1 (female football player). The qualitative findings for the simulated jump data were similar to those for the experimental jump data. 

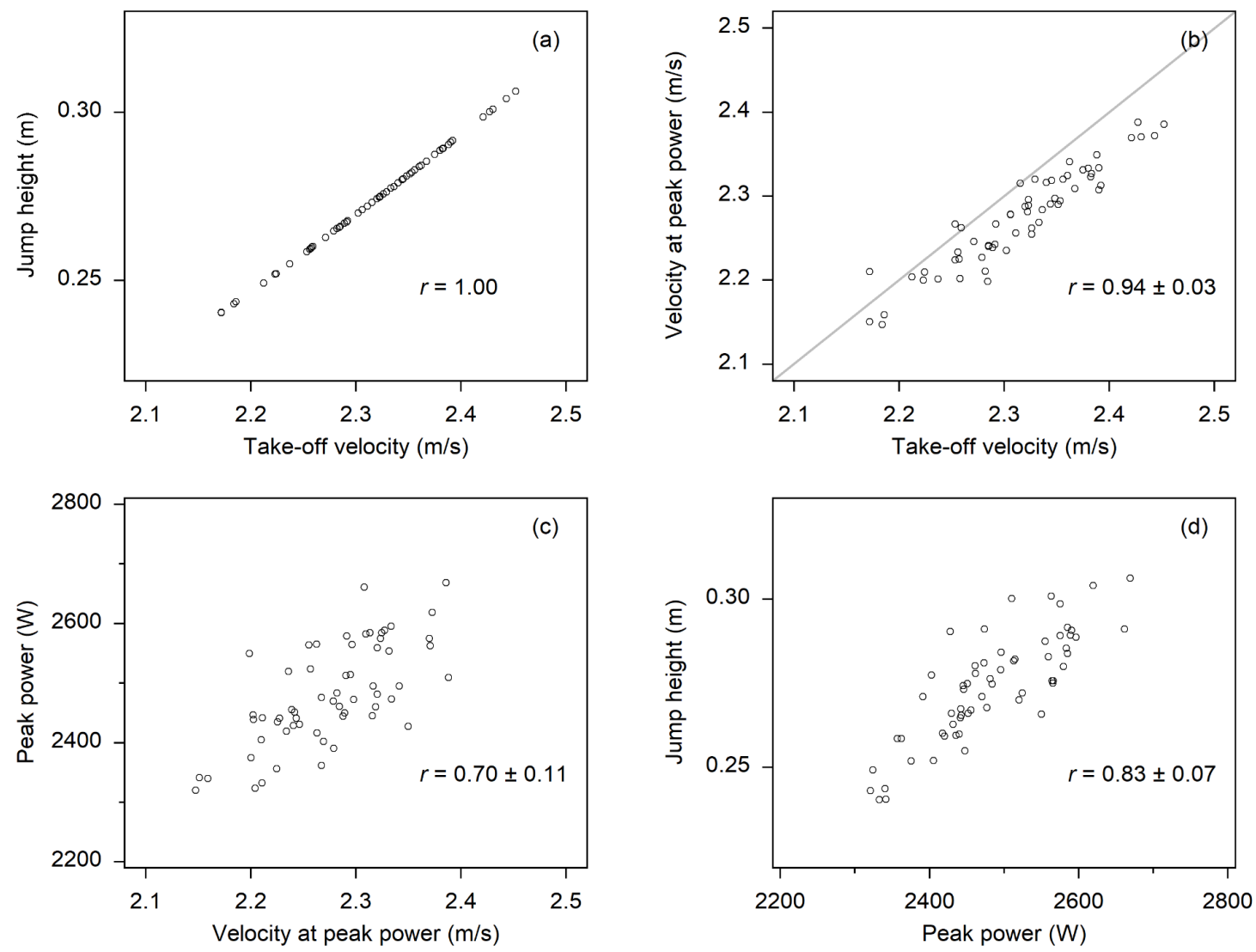

Figure 5. Plots to illustrate the biomechanical link between jump height and peak power $(r \pm 90 \% \mathrm{CI})$ : (a) Jump height is exactly determined by the jumper's take-off velocity. (b) Take-off velocity is very nearly equal to the velocity at peak power. Variability in this relationship might be due to differences in the timing of the proximal-distal extension of the hip, knee, and ankle joints toward the end of the upward propulsive phase. (c) Peak power is strongly related to the velocity at peak power (because power is the product of force and velocity). Variability in this relationship might be due to differences in the magnitude of the ground reaction force due to differences in countermovement depth or the rate of countermovement. (d) Therefore, jump height is strongly related to peak power. Data for Participant 1. 